\title{
Symbolic computation of hyperbolic tangent solutions for nonlinear differential-difference equations *
}

\author{
D. Baldwin ${ }^{a} \ddot{U}$. Göktaş ${ }^{b}$ W. Hereman ${ }^{a, c, *}$ \\ ${ }^{a}$ Department of Mathematical and Computer Sciences, Colorado School of Mines, \\ Golden, CO 80401-1887, U.S.A. \\ ${ }^{\mathrm{b}}$ Wolfram Research, Inc., 100 Trade Center Drive, Champaign, IL 61820, U.S.A. \\ ${ }^{\mathrm{c}}$ Department of Applied Mathematics, University of Stellenbosch, Private Bag X1, \\ 7602 Matieland, South Africa
}

\begin{abstract}
A new algorithm is presented to find exact traveling wave solutions of differentialdifference equations in terms of tanh functions. For systems with parameters, the algorithm determines the conditions on the parameters so that the equations might admit polynomial solutions in tanh.

Examples illustrate the key steps of the algorithm. Parallels are drawn through discussion and example to the tanh-method for partial differential equations.

The new algorithm is implemented in Mathematica. The package DDESpecialSolutions.m can be used to automatically compute traveling wave solutions of nonlinear polynomial differential-difference equations. Use of the package, implementation issues, scope, and limitations of the software are addressed.
\end{abstract}

\section{Program summary}

Title of program: DDESpecialSolutions.m

Catalogue identifier (supplied by the Publisher):

Distribution format (supplied by the Program Library):

Computers: Created using a PC, but can be run on UNIX and Apple machines

Operating systems under which the program has been tested: Windows 2000 and XP

Programming language used: Mathematica

Memory required to execute with typical data: $9 \mathrm{MB}$

Number of processors used: 1

Has the code been vectorised or parallelized?: No

Number of bytes in distributed program, including test data, etc.: 104761

Nature of physical problem: The program computes exact solutions to differentialdifference equations in terms of the tanh function. Such solutions describe particle vibrations in lattices, currents in electrical networks, pulses in biological chains, etc. Method of solution: After the differential-difference equation is placed in a traveling frame of reference, the coefficients of a candidate polynomial solution in tanh

Preprint submitted to Computer Physics Communications 13 August 2018 
are solved for. The resulting solution is tested by substitution into the original differential-difference equation.

Restrictions on the complexity of the program: The system of differential-difference equations must be polynomial. Solutions are polynomial in tanh.

Typical running time: The average run time of 16 cases (such as Toda, Volterra, and Ablowitz-Ladik lattices) is 0.228 seconds with a standard deviation of 0.165 seconds on a $2.4 \mathrm{GHz}$ Pentium 4 with 512 MB RAM running Mathematica 4.1. The running time may vary considerably, depending on the complexity of the problem.

Key words: Exact solutions, traveling wave solutions, differential-difference equations, semi-discrete lattices, tanh-method

PACS: 02.70.Wz; 02.30.Ik; 02.30.Jr; 02.90.+p

\section{Introduction}

Since the work of Fermi, Pasta, and Ulam in the 1950s [14], differentialdifference equations (DDEs) have been the focus of many nonlinear studies (for references see e.g. [16,39]). There is renewed interest in DDEs, which can be used to model such physical phenomena as particle vibrations in lattices, currents in electrical networks, pulses in biological chains, etc. Unlike difference equations which are fully discretized, DDEs are semi-discretized with some (or all) of their spacial variables discretized while time is usually kept continuous. DDEs also play an important role in numerical simulations of nonlinear partial differential equations (PDEs), queuing problems, and discretizations in solid state and quantum physics.

There is a vast body of work on DDEs, including investigations of integrability criteria, the computation of densities, generalized and master symmetries, and recursion operators [15]. Notable is the work by Levi and colleagues [20,21], Yamilov [41,42] and co-workers [4,9,10,29,30,38], where the classification of DDEs (into canonical forms), integrability tests, and connections between integrable PDEs and DDEs are analyzed in detail. To a large extent, the classification and integrability testing of discrete equations parallels continuous equations (for reviews and references consult $[5,25,26,31]$ ).

A wealth of information about integrable DDEs can be found in papers by

\footnotetext{
* This material is based upon research supported by the National Science Foundation under Grants Nos. CCR-9901929, DMS-9732069 and DMS-9912293.

* Corresponding author.

Email address: whereman@mines.edu (W. Hereman).

URL: http://www.mines.edu/fs_home/whereman (W. Hereman).
} 
Suris $[32,33,34,35,36]$ and his book [37] in progress. Suris and others have shown that many lattices are closely related to the celebrated Toda lattice [40], its relativistic counterpart due to Ruijsenaars [28], the KvM lattice [18], and the two-component Volterra system $[29,30]$.

Recently, variants of the tanh-method have been successfully applied to many nonlinear polynomial systems of PDEs in any number of independent variables [6,11,12,13,27]. Baldwin et al. [7] implemented the tanh- method (and also sech, cn, and sn-methods) in Mathematica. Liu and Li implemented the tanhmethod [22,23] in Maple.

While there has been considerable work done on finding exact solutions to PDEs, as far as we could verify, little work is being done to symbolically compute exact solutions of DDEs. In this paper we present an adaptation of the tanh-method to solve nonlinear polynomial differential-difference equations, which to our knowledge is novel. Our algorithm applies to semi-discrete lattices and allows one to find closed form solutions that are polynomial in tanh.

Although the tanh method is easy to apply, it leads to fairly cumbersome algebra without guarantee that a tanh solution can be found [6]. We therefore present [8] the fully automated software package, DDESpecialSolutions.m in Mathematica, which implements the algorithm. Without intervention from the user, our software computes traveling wave solutions as polynomials in $T_{\mathbf{n}}=\tanh \xi_{\mathbf{n}}$, where $\xi_{\mathbf{n}}=\sum_{i=1}^{Q} d_{i} n_{i}+\sum_{i=j}^{N} c_{j} x_{j}+\delta$. The continuous variables $x_{i}$ and the discrete variables $n_{j}$ are combined with constants $c_{i}$ and $d_{j}$, and $\delta$ is the phase constant.

For systems of DDEs involving constant parameters (denoted by lower-case Greek letters), the software automatically determines the conditions on the parameters so that the given equations might admit polynomial solutions involving tanh. Obviously, since $\operatorname{sech}^{2} \xi=1-\tanh ^{2} \xi$ our code can find solutions in even powers of sech. But our code can not find solutions involving odd powers of sech.

The paper is organized as follows: Section 2 gives a discussion of the tanhmethod for PDEs with a worked example of a two-dimensional nonlinear PDE. Since the tanh-method for semi-discrete lattices so closely parallels the tanhmethod for evolutions equations, we believe that a discussion of the continuous case increases the transparency of the more complicated semi-discrete case. In Section 3 we present our tanh-method for DDEs with a worked example of a two-dimensional Toda equation followed by almost a dozen additional examples. A description of our Mathematica package, DDESpecialSolutions.m, is given in Section 4. We discuss our results and draw some conclusions in Section 5. Appendix A gives the full input and output of a test case. 


\section{Traveling Wave Solutions of PDEs}

In this section we discuss the tanh-method as it applies to systems of $P$ polynomial differential equations,

$$
\Delta\left(\mathbf{u}(\mathbf{x}), \mathbf{u}^{\prime}(\mathbf{x}), \mathbf{u}^{\prime \prime}(\mathbf{x}), \cdots, \mathbf{u}^{(m)}(\mathbf{x})\right)=\mathbf{0}
$$

where the dependent variable $\mathbf{u}$ has $P$ components $u_{i}$, the independent variable $\mathbf{x}$ has $N$ components $x_{j}$, and $\mathbf{u}^{(m)}(\mathbf{x})$ denotes the collection of mixed derivative terms of order $m$. We assume that any arbitrary coefficients parameterizing the system are strictly positive and denoted by lower-case Greek letters. To simplify the notation in the examples, we will write $u, v, w, \ldots$ instead of $u_{1}, u_{2}, u_{3}, \ldots$ and $x, y, t, \ldots$ instead of $x_{1}, x_{2}, x_{3}$, etc.

\subsection{Algorithm of the tanh-method for PDEs}

STEP 1 (Transform the PDE into a nonlinear ODE). We seek solutions in the traveling frame of reference,

$$
\xi=\sum_{j=1}^{N} c_{j} x_{j}+\delta
$$

where the components $c_{j}$ of the wave vector $\mathbf{x}$ and the phase $\delta$ are constants.

In the tanh method, we seek polynomial solutions expressible in hyperbolic tangent, $T=\tanh \xi$. Based on the identity $\cosh ^{2} \xi-\sinh ^{2} \xi=1$,

$$
\begin{aligned}
\tanh ^{\prime} \xi & =\operatorname{sech}^{2} \xi=1-\tanh ^{2} \xi, \\
\tanh ^{\prime \prime} \xi & =-2 \tanh \xi+2 \tanh ^{3} \xi, \text { etc. }
\end{aligned}
$$

Therefore, the first and consequently all higher-order derivatives are polynomial in $T$. Thus, repeatedly applying the chain rule,

$$
\frac{\partial \bullet}{\partial x_{j}}=\frac{\partial \xi}{\partial x_{j}} \frac{d T}{d \xi} \frac{d \bullet}{d T}=c_{j}\left(1-T^{2}\right) \frac{d \bullet}{d T}
$$

transforms (1) into a coupled system of nonlinear ODEs,

$$
\boldsymbol{\Gamma}\left(T, \mathbf{U}(T), \mathbf{U}^{\prime}(T), \ldots\right)=\mathbf{0},
$$

where $U_{i}(T)$ corresponds to $u_{i}(\mathbf{x})$.

STEP 2 (Determine the degree of the polynomial solutions). Since we seek 
polynomial solutions

$$
U_{i}(T)=\sum_{j=0}^{M_{i}} a_{i j} T^{j},
$$

the leading exponents $M_{i}$ must be determined before the $a_{i j}$ can be computed.

Substituting $U_{i}(T)$ into (6), the coefficients of every power of $T$ in every equation must vanish. In particular, the highest degree terms must vanish. Since the highest degree terms only depend on $T^{M_{i}}$ in (7), it suffices to substitute $U_{i}(T)=T^{M_{i}}$ into (6). In the resulting polynomial system $\mathbf{P}(T)=0$, equating every two possible highest exponents in every component $P_{i}$ gives a linear system for determining the $M_{i}$. The linear system is then solved for $M_{i}$.

If one or more exponents $M_{i}$ remain undetermined, we assign a strictly positive integer value to the free $M_{i}$, so that every equation in (6) has at least two different terms with equal highest exponents in $T$.

STEP 3 (Derive the algebraic system for the coefficients $a_{i j}$ ). To generate the system for the unknown coefficients $a_{i j}$ and wave parameters $c_{j}$, substitute (7) into (6) and set the coefficients of $T^{j}$ to zero. The resulting nonlinear algebraic system for the unknown $a_{i j}$ is parameterized by the wave parameters $c_{j}$ and the parameters in (1), if any.

STEP 4 (Solve the nonlinear parameterized algebraic system). The most difficult step of the method is analyzing and solving the nonlinear algebraic system. To solve the system we designed a customized, yet powerful, nonlinear solver.

The nonlinear algebraic system is solved with the following assumptions:

- all parameters (lower-case Greek letters) in (1) are strictly positive. (Vanishing parameters may change the exponents $M_{i}$ in Step 2).

- the coefficients of the highest power terms $\left(a_{i M_{i}}, i=1, \cdots, P\right)$ in $(7)$ are all nonzero (for consistency with Step 2); and,

- all $c_{j}$ are nonzero (demanded by the nature of the solutions we seek).

The algebraic system is solved recursively, starting with the simplest equation, and continually back-substituting solutions. This process is repeated until the system is completely solved.

To guide the recursive process, we designed functions to (i) factor, split, and simplify the equations; (ii) sort the equations according to their complexity; (iii) solve the equations for sorted unknowns; (iv) substitute solutions into the remaining equations; and (v) collect the solution branches and constraints.

This strategy is similar to what one would do by hand. If there are numerous parameters in the system or if it is of high degree, there is no guarantee that 
our solver will return a suitable result, let alone a complete result.

STEP 5 (Build and test solutions). Substituting the solutions from Step 4 into (7) and reversing Step 1, one obtains the explicit solutions in the original variables. It is prudent to test the solutions by substituting them into (1).

\subsection{Application of the tanh-method to a PDE}

Consider the $(2+1)$ dispersive long wave system [24],

$$
\left\{\begin{aligned}
u_{y t}+v_{x x}+u_{x} u_{y}+u u_{x y} & =0 \\
v_{t}+u_{x}+u_{x x y}+u_{x} v+u v_{x} & =0
\end{aligned}\right.
$$

which is related to the Eckhaus system.

Applying chain rule (5) repeatedly to (8), we get the coupled ODEs,

$$
\begin{gathered}
2 c_{2} T\left(c_{3}+c_{1} U\right) U^{\prime}+c_{1} c_{2}\left(T^{2}-1\right) U^{2}+2 c_{1}^{2} T V^{\prime} \\
+\left(T^{2}-1\right)\left(c_{2}\left(c_{3}+c_{1} U\right) U^{\prime \prime}+c_{1}^{2} V^{\prime \prime}\right)=0 \\
c_{1}\left(1+2\left(3 T^{2}-1\right) c_{1} c_{2}+V\right) U^{\prime}+\left(c_{3}+c_{1} U\right) V^{\prime} \\
+c_{1}^{2} c_{2}\left(T^{2}-1\right)\left(6 T U^{\prime \prime}+\left(T^{2}-1\right) U^{\prime \prime \prime}\right)=0
\end{gathered}
$$

where $T=\tanh \left(c_{1} x+c_{2} y+c_{3} t+\delta\right)$.

To compute the degree of the polynomial solution(s), substitute $U(T)=T^{M_{1}}$ and $V(T)=T^{M_{2}}$ into (9) and pull off the exponents of $T$ (see Table 1 ). Removing non-dominant exponents and equating possible highest exponents,

\begin{tabular}{c|ccc|c} 
Term & Exponents of $T$ \\
\hline \hline$u_{y t}$ & $\left\{M_{1}-2, M_{1}\right\}$ \\
\hline$v_{x x}$ & $\left\{M_{2}-2, M_{2}\right\}$ \\
\hline$u_{x} u_{y}, u u_{x y}$ & $\left\{2 M_{1}, 2 M_{1}-2\right\}$ & & Term & Exponents of $T$ \\
\hline$v_{t}$ & $\left\{M_{2}-1\right\}$ \\
\hline$u_{x x y}$ & $\left\{M_{1}-3, M_{1}-1, M_{1}+1\right\}$ \\
\hline$u_{x} v, u v_{x}$ & $\left\{M_{1}+M_{2}-1\right\}$
\end{tabular}

Table 1

The exponents of $T$ in (9) after substituting $U_{i}(T)=T^{M_{i}}$

we find $M_{1}=M_{2}$ (from $u_{y t}$ and $v_{x x}$ ) or $2 M_{1}=M_{2}$ (from $u_{x} u_{y}$ or $u u_{x y}$ and $\left.v_{x x}\right)$ from the first equation. Then, from the second equation, we find $M_{2}=2$ 
(from $u_{x} v$ or $u v_{x}$ and $u_{x x y}$ ). This gives us two branches,

$$
\begin{aligned}
& \left\{\begin{array}{l}
M_{1}=1, \quad U(T)=a_{10}+a_{11} T, \\
M_{2}=2, \quad V(T)=a_{20}+a_{21} T+a_{22} T^{2},
\end{array}\right. \\
& \begin{cases}M_{1}=2, & U(T)=a_{10}+a_{11} T+a_{12} T^{2}, \\
M_{2}=2, & V(T)=a_{20}+a_{21} T+a_{22} T^{2} .\end{cases}
\end{aligned}
$$

For the first branch, substituting (10) into (9) and equating the coefficients of $T^{j}$ to zero gives

$$
\begin{aligned}
c_{1}\left(2 a_{22} c_{1}+a_{11}^{2} c_{2}\right) & =0, \\
c_{1}\left(2 a_{22} c_{1}+a_{11}^{2} c_{2}\right) & =0, \\
a_{21} c_{1}^{2}+a_{10} a_{11} c_{1} c_{2}+a_{11} c_{2} c_{3} & =0, \\
a_{11} c_{1}\left(a_{22}+2 c_{1} c_{2}\right) & =0, \\
a_{11} c_{1}+a_{11} a_{20} c_{1}+a_{10} a_{21} c_{1}-2 a_{11} c_{1}^{2} c_{2}+a_{21} c_{3} & =0, \\
a_{11} a_{21} c_{1}+a_{10} a_{22} c_{1}+a_{22} c_{3} & =0 .
\end{aligned}
$$

Similarly, for the second branch, substituting (11) into (9) and setting the coefficients of $T^{j}$ to zero yields

$$
\begin{aligned}
a_{11} a_{12} c_{1} c_{2} & =0, \\
a_{12}^{2} c_{1} c_{2} & =0, \\
a_{21} c_{1}^{2}+a_{10} a_{11} c_{1} c_{2}-3 a_{11} a_{12} c_{1} c_{2}+a_{11} c_{2} c_{3} & =0, \\
2 a_{22} c_{1}^{2}+a_{11}^{2} c_{1} c_{2}+2 a_{10} a_{12} c_{1} c_{2}-2 a_{12}^{2} c_{1} c_{2}+2 a_{12} c_{2} c_{3} & =0, \\
2 a_{22} c_{1}^{2}+a_{11}^{2} c_{1} c_{2}+2 a_{10} a_{12} c_{1} c_{2}+2 a_{12} c_{2} c_{3} & =0, \\
c_{1}\left(a_{12} a_{21}+a_{11} a_{22}+2 a_{11} c_{1} c_{2}\right) & =0, \\
a_{12} c_{1}\left(a_{22}+6 c_{1} c_{2}\right) & =0, \\
a_{11} c_{1}+a_{11} a_{20} c_{1}+a_{10} a_{21} c_{1}-2 a_{11} c_{1}^{2} c_{2}+a_{21} c_{3} & =0, \\
a_{12} c_{1}+a_{12} a_{20} c_{1}+a_{11} a_{21} c_{1}+a_{10} a_{22} c_{1}-8 a_{12} c_{1}^{2} c_{2}+a_{22} c_{3} & =0 .
\end{aligned}
$$

For the first branch, solving (12) under the assumption that $a_{11}, a_{22}, c_{1}, c_{2}$, and $c_{3}$ are nonzero, we find

$$
\begin{gathered}
a_{10}=-\frac{c_{3}}{c_{1}}, \quad a_{11}= \pm 2 c_{1}, \\
a_{20}=2 c_{1} c_{2}-1, \quad a_{21}=0, \quad a_{22}=-2 c_{1} c_{2} .
\end{gathered}
$$

Substituting (14) into (10) and returning to $u(x, y, t)$ and $v(x, y, t)$, we get

$$
\begin{aligned}
& u(x, y, t)=-\frac{c_{3}}{c_{1}} \pm 2 c_{1} \tanh \left(c_{1} x+c_{2} y+c_{3} t+\delta\right), \\
& v(x, y, t)=2 c_{1} c_{2}-1-2 c_{1} c_{2} \tanh ^{2}\left(c_{1} x+c_{2} y+c_{3} t+\delta\right) .
\end{aligned}
$$

In the second branch, the equation $a_{12}^{2} c_{1} c_{2}=0$ in (13) is inconsistent with our 
assumption that $a_{12}, a_{22}, c_{1}, c_{2}$, and $c_{3}$ are nonzero. This branch does not yield a solution to (8).

Substituting (15) into (8), we verify that our solution does indeed satisfy the original system.

\section{Tanh method for nonlinear DDEs}

The tanh-method can be adapted to solve nonlinear polynomial DDEs. Apart from slight, yet important modifications, the steps mirror those in Section 2.

Given is a system of $M$ polynomial DDEs,

$$
\begin{aligned}
& \Delta\left(\mathbf{u}_{\mathbf{n}+\mathbf{p}_{1}}(\mathbf{x}), \mathbf{u}_{\mathbf{n}+\mathbf{p}_{2}}(\mathbf{x}), \ldots, \mathbf{u}_{\mathbf{n}+\mathbf{p}_{k}}(\mathbf{x})\right. \\
& \mathbf{u}_{\mathbf{n}+\mathbf{p}_{1}}^{\prime}(\mathbf{x}), \mathbf{u}_{\mathbf{n}+\mathbf{p}_{2}}^{\prime}(\mathbf{x}), \ldots, \mathbf{u}_{\mathbf{n}+\mathbf{p}_{k}}^{\prime}(\mathbf{x}), \ldots, \\
&\left.\quad \mathbf{u}_{\mathbf{n}+\mathbf{p}_{1}}^{(r)}(\mathbf{x}), \mathbf{u}_{\mathbf{n}+\mathbf{p}_{2}}^{(r)}(\mathbf{x}), \ldots, \mathbf{u}_{\mathbf{n}+\mathbf{p}_{k}}^{(r)}(\mathbf{x})\right)=\mathbf{0}
\end{aligned}
$$

where the dependent variable $\mathbf{u}$ has $M$ components $u_{i}$, the continuous variable $\mathbf{x}$ has $N$ components $x_{i}$, the discrete variable $\mathbf{n}$ has $Q$ components $n_{j}$, the $k$ shift vectors $\mathbf{p}_{i} \in \mathbb{Z}^{Q}$, and $\mathbf{u}^{(r)}(\mathbf{x})$ denotes the collection of mixed derivative terms of order $r$. We assume that any arbitrary coefficients that parameterize the system are strictly positive and denoted by lower-case Greek letters.

To simplify notation in the examples, we use dependent variables $u, v, w, \ldots$ instead of $u_{1}, u_{2}, u_{3}, \ldots$, continuous independent variables $x, y, t, \ldots$ instead of $x_{1}, x_{2}, x_{3}, \ldots$, and lattice points $n, m, \ldots$, instead of $n_{1}, n_{2}$, etc. For example, the two-component Volterra equation [34],

$$
\begin{aligned}
& \dot{u}_{n}=u_{n}\left(v_{n}-v_{n-1}\right) \\
& \dot{v}_{n}=v_{n}\left(u_{n+1}-u_{n}\right),
\end{aligned}
$$

has $\mathbf{u}=\left(u_{1}, u_{2}\right)=(u, v), \mathbf{x}=x_{1}=t, \mathbf{n}=n_{1}=n$, and $\mathbf{p}_{1}=p_{1}=-1$, $\mathbf{p}_{2}=p_{2}=0, \mathbf{p}_{3}=p_{3}=1$.

\subsection{Algorithm of the tanh-method for DDEs}

STEP 1 (Transform the DDE into a nonlinear DDE in T). We seek solutions in the traveling frame of reference,

$$
\xi_{\mathbf{n}}=\sum_{i=1}^{Q} d_{i} n_{i}+\sum_{j=1}^{N} c_{j} x_{j}+\delta=\mathbf{d} \cdot \mathbf{n}+\mathbf{c} \cdot \mathbf{x}+\delta,
$$


where the coefficients $c_{1}, c_{2}, \ldots, c_{N}, d_{1}, d_{2}, \ldots, d_{Q}$ and the phase $\delta$ are all constants. The dot $(\cdot)$ denotes the Euclidean inner product.

Using the properties of hyperbolic tangent, $T_{\mathbf{n}}=\tanh \xi_{\mathbf{n}}$, repeatedly applying the chain rule,

$$
\frac{d \bullet}{d x_{j}}=\frac{\partial \xi_{\mathbf{n}}}{\partial x_{j}} \frac{d T_{\mathbf{n}}}{d \xi_{\mathbf{n}}} \frac{d \bullet}{d T_{\mathbf{n}}}=c_{j}\left(1-T_{\mathbf{n}}^{2}\right) \frac{d \bullet}{d T_{\mathbf{n}}}
$$

transforms (16) into

$$
\begin{aligned}
\Delta\left(\mathbf{U}_{\mathbf{n}+\mathbf{p}_{1}}\left(T_{\mathbf{n}}\right), \ldots, \mathbf{U}_{\mathbf{n}+\mathbf{p}_{k}}\left(T_{\mathbf{n}}\right), \mathbf{U}_{\mathbf{n}+\mathbf{p}_{1}}^{\prime}\left(T_{\mathbf{n}}\right), \ldots, \mathbf{U}_{\mathbf{n}+\mathbf{p}_{k}}^{\prime}\left(T_{\mathbf{n}}\right)\right. \\
\left.\ldots, \mathbf{U}_{\mathbf{n}+\mathbf{p}_{1}}^{(r)}\left(T_{\mathbf{n}}\right), \ldots, \mathbf{U}_{\mathbf{n}+\mathbf{p}_{k}}^{(r)}\left(T_{\mathbf{n}}\right)\right)=\mathbf{0 .}
\end{aligned}
$$

It is important to note that for any $s(s=1, \cdots, k), \mathbf{U}_{\mathbf{n}+\mathbf{p}_{s}}$ is a function of $T_{\mathbf{n}}$ and not $T_{\mathbf{n}+\mathbf{p}_{s}}$. Using the identity,

$$
\tanh (x+y)=\frac{\tanh x+\tanh y}{1+\tanh x \tanh y}
$$

we can write

$$
T_{\mathbf{n}+\mathbf{p}_{s}}=\frac{T_{\mathbf{n}}+\tanh \phi_{s}}{1+T_{\mathbf{n}} \tanh \phi_{s}}
$$

where

$$
\phi_{s}=\mathbf{p}_{s} \cdot \mathbf{d}=p_{s 1} d_{1}+p_{s 2} d_{2}+\cdots+p_{s Q} d_{Q},
$$

and $p_{s j}$ is the $j$-th component of shift vector $\mathbf{p}_{s}$.

STEP 2 (Determine the degree of the polynomial solutions). Seeking solutions of the form

$$
U_{i, \mathbf{n}}\left(T_{\mathbf{n}}\right)=\sum_{j=0}^{M_{i}} a_{i j} T_{\mathbf{n}}^{j}
$$

we must first compute the leading exponents $M_{i}$. As in the continuous case, we can do this by substituting only the leading term,

$$
U_{i, \mathbf{n}+\mathbf{p}_{s}}\left(T_{\mathbf{n}}\right)=T_{\mathbf{n}+\mathbf{p}_{s}}^{M_{i}}=\left[\frac{T_{\mathbf{n}}+\tanh \phi_{s}}{1+T_{\mathbf{n}} \tanh \phi_{s}}\right]^{M_{i}},
$$

with $\phi_{s}$ in $(23)$.

Suppose we are interested in balancing terms with shift $\mathbf{p}_{l}$, then terms with shifts other than $\mathbf{p}_{l}$, say $\mathbf{p}_{s}$, will not effect the balance since $U_{i, \mathbf{n}+\mathbf{p}_{s}}$ can be interpreted as being of degree zero in $T_{\mathbf{n}+\mathbf{p}_{l}}$. For instance, if $\mathbf{p}_{l}=\mathbf{0}$, then $U_{i, \mathbf{n}+\mathbf{0}}\left(T_{\mathbf{n}}\right)=T_{\mathbf{n}}^{M_{i}}$ is of degree $M_{i}$ in $T_{\mathbf{n}}$ and $U_{i, \mathbf{n}+\mathbf{p}_{s}}(T)=\left[\frac{T_{\mathbf{n}}+\tanh \phi_{s}}{1+T_{\mathbf{n}} \tanh \phi_{s}}\right]^{M_{i}}$ is of degree zero in $T_{\mathbf{n}}$. 
Therefore, if we need to balance terms with shift $\mathbf{p}_{l}$, we substitute

$$
U_{i, \mathbf{n}+\mathbf{p}_{s}}= \begin{cases}\chi_{i} T_{\mathbf{n}}^{M_{i}}, & s=l \\ \chi_{i}, & s \neq l\end{cases}
$$

into (20) and proceed as in Step 2 of Section 2. We then continue with the union of the solutions found for $l=1,2, \ldots, k$.

STEP 3 (Derive the algebraic system for the coefficients $a_{i j}$ ). Substitute

$$
U_{i, \mathbf{n}+\mathbf{p}_{s}}\left(T_{\mathbf{n}}\right)=\sum_{j=0}^{M_{i}} a_{i j} T_{\mathbf{n}+\mathbf{p}_{s}}^{j}=\sum_{j=0}^{M_{i}} a_{i j}\left[\frac{T_{\mathbf{n}}+\tanh \phi_{s}}{1+T_{\mathbf{n}} \tanh \phi_{s}}\right]^{j},
$$

into (20), with $\phi_{s}$ in (23). Applying (21) one can split tanh $\phi_{s}$ into powers of $\tanh d_{i}$. While doing so, we repeatedly clear the denominators. The resulting nonlinear algebraic system for the unknowns $a_{i j}$ is parameterized by $c_{1}, c_{2}, \ldots$, $c_{N}, \tanh \left(d_{1}\right), \tanh \left(d_{2}\right), \ldots, \tanh \left(d_{Q}\right)$ and any parameters (lower-case Greek letters) in (16).

STEP 4 (Solve the nonlinear parameterized system). This step is the same as in the continuous case; we solve the system for $a_{i j}$ in terms of the parameters $c_{1}, c_{2}, \ldots, c_{N}, \tanh \left(d_{1}\right), \tanh \left(d_{2}\right), \ldots, \tanh \left(d_{Q}\right)$ and any parameters (lower-case Greek letters) in (16).

STEP 5 (Build and test the solitary wave solutions). Substitute the solution found in Step 4 into (24) and reverse Step 1. Then, test the solutions (in the original variables) by substitution into (16).

\subsection{Example of a differential-difference equation}

To illustrate the method, we derive an exact solution of the $(2+1)$-dimensional Toda lattice [19],

$$
\frac{\partial^{2} y_{n}}{\partial x \partial t}=\exp \left(y_{n-1}-y_{n}\right)-\exp \left(y_{n}-y_{n+1}\right)
$$

where $y_{n}(x, t)$ is the displacement from equilibrium of the $n$-th unit mass under an exponential decaying interaction force between nearest neighbors. To write (28) as a polynomial DDE, set

$$
\frac{\partial u_{n}}{\partial t}=\exp \left(y_{n-1}-y_{n}\right)-1
$$


Then,

$$
\exp \left(y_{n-1}-y_{n}\right)=\frac{\partial u_{n}}{\partial t}+1
$$

and (28) becomes

$$
\frac{\partial^{2} y_{n}}{\partial x \partial t}=\frac{\partial u_{n}}{\partial t}+1-\left(\frac{\partial u_{n+1}}{\partial t}+1\right)=\frac{\partial u_{n}}{\partial t}-\frac{\partial u_{n+1}}{\partial t}
$$

Integrating (31) and ignoring the integration constant, we find

$$
\frac{\partial y_{n}}{\partial x}=u_{n}-u_{n+1}
$$

Differentiating (29) with respect to $x$ and using (30) and (32), we compute

$$
\begin{aligned}
\frac{\partial^{2} u_{n}}{\partial x \partial t} & =\frac{\partial}{\partial x}\left(\exp \left(y_{n-1}-y_{n}\right)-1\right) \\
& =\exp \left(y_{n-1}-y_{n}\right)\left(\frac{\partial y_{n-1}}{\partial x}-\frac{\partial y_{n}}{\partial x}\right) \\
& =\left(\frac{\partial u_{n}}{\partial t}+1\right)\left[\left(u_{n-1}-u_{n}\right)-\left(u_{n}-u_{n+1}\right)\right] \\
& =\left(\frac{\partial u_{n}}{\partial t}+1\right)\left(u_{n-1}-2 u_{n}+u_{n+1}\right)
\end{aligned}
$$

So, (28) can be replaced by the polynomial equation (36).

By repeatedly applying the chain rule (19) to (36), one gets

$$
\begin{aligned}
c_{1} c_{2}\left(1-T_{n}^{2}\right)\left[2 T_{n} U_{n}^{\prime}-\left(1-T_{n}^{2}\right) U_{n}^{\prime \prime}\right]+ & \\
& {\left[1+c_{1}\left(1-T_{n}^{2}\right) U_{n}^{\prime}\right]\left[U_{n-1}-2 U_{n}+U_{n+1}\right]=0 . }
\end{aligned}
$$

where $T_{n}=\tanh \left(d_{1} n+c_{1} x+c_{2} t+\delta\right)$.

For this system, we have three shifts $p_{1}=-1, p_{2}=0$, and $p_{3}=1$. Substituting (26) into (37) and pulling off the highest exponents, we find $\left\{M_{1}\right\}$ for shift $p_{1}$ and $p_{3}$, and $\left\{M_{1}, M_{1}+1, M_{1}+2,2 M_{1}+1\right\}$ for shift $p_{2}$. With only one term, neither shifts $p_{1}$ or $p_{3}$ contribute any solutions. Equating the two highest terms from the shift $p_{2}, M_{1}+2$ and $2 M_{1}+1$, we find $M_{1}=1$.

Substituting (27) into (37), clearing the denominator and setting coefficients of power terms in $T_{n}$ to zero, gives

$$
\begin{aligned}
a_{11}-c_{1} & =0, \\
c_{1} c_{2}-\tanh ^{2}\left(d_{1}\right)-a_{11} c_{2} \tanh ^{2}\left(d_{1}\right) & =0 \\
c_{1} c_{2}-\tanh ^{2}\left(d_{1}\right)-2 a_{11} c_{2} \tanh ^{2}\left(d_{1}\right)+c_{1} c_{2} \tanh ^{2}\left(d_{1}\right) & =0 .
\end{aligned}
$$


Assuming $d_{1}, c_{1}$, and $a_{11}$ nonzero, the solution of (38) is

$$
a_{10}=\text { arbitrary, } \quad a_{11}=c_{1}=\frac{\sinh ^{2}\left(d_{1}\right)}{c_{2}} .
$$

Then, the closed form solution of (36) is

$$
u_{n}(x, t)=a_{10}+\frac{1}{c_{2}} \sinh ^{2}\left(d_{1}\right) \tanh \left[d_{1} n+\frac{\sinh ^{2}\left(d_{1}\right)}{c_{2}} x+c_{2} t+\delta\right]
$$

where $a_{10}, d_{1}, c_{2}$ and $\delta$ are arbitrary. The algorithm must be repeated if any of the parameters in (16) are set to zero.

\subsection{Further examples}

We applied the tanh algorithm to solve a variety of nonlinear lattice equations in $(1+1)$-dimensions. The results are summarized in Table 2. For notational simplicity, we denote $\frac{\partial u_{n}}{\partial t}$ by $\dot{u}_{n}$.

The Ablowitz-Ladik equation [1,2] is a discretization of the nonlinear Schrödinger equation. The Toda lattices $[32,34,40]$ describe vibrations in mass-spring lattices with an exponential interaction force. The Volterra type equations $[3,17,34]$ are discretizations of the Korteweg-de Vries (KdV) and modified KdV equations.

\section{Description of DDESpecialSolutions.m}

The format of DDESpecialSolutions is similar to the Mathematica function DSolve. The output is a list of sub-lists with solutions and constraints. The Backus-Naur form of the function is:

$$
\begin{aligned}
\langle\text { Main Function }\rangle \rightarrow & \text { DDESpecialSolutions }[\langle\text { Equations }\rangle,\langle\text { Functions }\rangle, \\
& \langle\text { Discrete Variables }\rangle,\langle\text { Continuous Variables }\rangle, \\
& \langle\text { Parameters }\rangle,\langle\text { Options }\rangle] \\
\langle\text { Options }\rangle \rightarrow & \text { Verbose } \rightarrow\langle\text { Bool }\rangle \mid \text { InputForm } \rightarrow\langle\text { Bool }\rangle \mid \\
& \text { DegreeOfThePolynomial } \rightarrow\langle\text { Listof Rules }\rangle \mid \\
& \text { NumericTest } \rightarrow\langle\text { Bool }\rangle \mid \text { SymbolicTest } \rightarrow\langle\text { Bool }\rangle \\
\langle\text { Bool }\rangle \rightarrow & \text { True } \mid \text { False } \\
\langle\text { List of Rules }\rangle \rightarrow & \{\mathrm{m}[1] \rightarrow \text { Integer, } \mathrm{m}[2] \rightarrow \text { Integer }, . . .\}
\end{aligned}
$$




\begin{tabular}{|c|c|}
\hline \multirow{2}{*}{$\begin{array}{l}\text { Equation Name } \\
\text { Reference }\end{array}$} & Equations \\
\hline & Solution(s) \\
\hline $\begin{array}{l}\text { Ablowitz-Ladik } \\
\text { Lattice }\end{array}$ & $\begin{array}{l}\dot{u}_{n}(t)=\left(\alpha+u_{n} v_{n}\right)\left(u_{n+1}+u_{n-1}\right)-2 \alpha u_{n} \\
\dot{v}_{n}(t)=-\left(\alpha+u_{n} v_{n}\left(v_{n+1}+v_{n-1}\right)+2 \alpha v_{n}\right.\end{array}$ \\
\hline$[1,2]$ & $\begin{array}{l}u_{n}(t)=\frac{\alpha \sinh ^{2}\left(d_{1}\right)}{a_{21}}\left( \pm 1-\tanh \left[d_{1} n+2 \alpha t \sinh ^{2}\left(d_{1}\right)+\delta\right]\right) \\
v_{n}(t)=a_{21}\left( \pm 1+\tanh \left[d_{1} n+2 \alpha \sinh ^{2}\left(d_{1}\right) t+\delta\right]\right)\end{array}$ \\
\hline Toda Lattice & $\ddot{u}_{n}(t)=\left(\dot{u}_{n}+1\right)\left(u_{n-1}-2 u_{n}+u_{n+1}\right)$ \\
\hline$[40]$ & $u_{n}(t)=a_{10} \pm \sinh \left(d_{1}\right) \tanh \left[d_{1} n \pm \sinh \left(d_{1}\right) t+\delta\right]$ \\
\hline $\begin{array}{l}(2+1) \text { Dimensional } \\
\text { Toda Lattice }\end{array}$ & $\frac{\partial^{2} u_{n}}{\partial x \partial t}(x, t)=\left(\frac{\partial u_{n}}{\partial t}+1\right)\left(u_{n-1}-2 u_{n}+u_{n+1}\right)$ \\
\hline [19] & $u_{n}(x, t)=a_{10}+\frac{1}{c_{2}} \sinh ^{2}\left(d_{1}\right) \tanh \left[d_{1} n+\frac{\sinh ^{2}\left(d_{1}\right)}{c_{2}} x+c_{2} t+\delta\right.$ \\
\hline $\begin{array}{l}\text { Another Toda } \\
\text { Lattice }\end{array}$ & $\begin{aligned} \dot{u}_{n}(t) & =u_{n}\left(v_{n}-v_{n-1}\right) \\
\dot{v}_{n}(t) & =v_{n}\left(u_{n+1}-u_{n}\right)\end{aligned}$ \\
\hline$[32]$ & $\begin{array}{l}u_{n}(t)=-\operatorname{coth}\left(d_{1}\right) c_{1}+c_{1} \tanh \left(d_{1} n+c_{1} t+\delta\right) \\
v_{n}(t)=-\operatorname{coth}\left(d_{1}\right) c_{1}-c_{1} \tanh \left(d_{1} n+c_{1} t+\delta\right)\end{array}$ \\
\hline $\begin{array}{l}\text { Relativistic Toda } \\
\text { Lattice }\end{array}$ & $\begin{array}{l}\dot{u}_{n}(t)=\left(1+\alpha u_{n}\right)\left(v_{n}-v_{n-1}\right) \\
\dot{v}_{n}(t)=v_{n}\left(u_{n+1}-u_{n}+\alpha v_{n+1}-\alpha v_{n-1}\right)\end{array}$ \\
\hline$[34]$ & $\begin{array}{l}u_{n}(t)=-\frac{1}{\alpha}-c_{1} \operatorname{coth}\left(d_{1}\right)+c_{1} \tanh \left(d_{1} n+c_{1} t+\delta\right) \\
v_{n}(t)=\frac{c_{1}}{\alpha} \operatorname{coth}\left(d_{1}\right)-\frac{c_{1}}{\alpha} \tanh \left(d_{1} n+c_{1} t+\delta\right)\end{array}$ \\
\hline $\begin{array}{l}\text { Another Relativistic } \\
\text { Toda Lattice }\end{array}$ & $\begin{aligned} \dot{u}_{n}(t) & =\left(u_{n+1}-v_{n}\right) v_{n}-\left(u_{n-1}-v_{n-1}\right) v_{n-1} \\
\dot{v}_{n}(t) & =v_{n}\left(u_{n+1}-u_{n}\right)\end{aligned}$ \\
\hline$[32]$ & $\begin{array}{l}u_{n}(t)=\operatorname{coth}\left(d_{1}\right) c_{1}+c_{1} \tanh \left(d_{1} n+c_{1} t+\delta\right) \\
v_{n}(t)=\operatorname{coth}\left(d_{1}\right) c_{1}+c_{1} \tanh \left(d_{1} n+c_{1} t+\delta\right)\end{array}$ \\
\hline Volterra Lattice & $\begin{aligned} \dot{u}_{n}(t) & =u_{n}\left(v_{n}-v_{n-1}\right) \\
\dot{v}_{n}(t) & =v_{n}\left(u_{n+1}-u_{n}\right)\end{aligned}$ \\
\hline$[34]$ & $\begin{array}{l}u_{n}(t)=-c_{1} \operatorname{coth}\left(d_{1}\right)+c_{1} \tanh \left(d_{1} n+c_{1} t+\delta\right) \\
v_{n}(t)=-c_{1} \operatorname{coth}\left(d_{1}\right)-c_{1} \tanh \left(d_{1} n+c_{1} t+\delta\right)\end{array}$ \\
\hline $\begin{array}{l}\text { Discretized mKdV } \\
\text { Lattice }\end{array}$ & $\dot{u}_{n}(t)=\left(\alpha-u_{n}^{2}\right)\left(u_{n+1}-u_{n-1}\right)$ \\
\hline$[3]$ & $u_{n}(t)= \pm \sqrt{\alpha} \tanh \left(d_{1}\right) \tanh \left[d_{1} n+2 \alpha \tanh \left(d_{1}\right) t+\delta\right]$ \\
\hline Hybrid Lattice & $\dot{u}_{n}(t)=\left(1+\alpha u_{n}+\beta u_{n}^{2}\right)\left(u_{n-1}-u_{n+1}\right)$ \\
\hline$[17]$ & $u_{n}(t)=\frac{-\alpha \pm \sqrt{\alpha^{2}-4 \beta \tanh \left(d_{1}\right)}}{2 \beta} \tanh \left[d_{1} n+\frac{\alpha^{2}-4 \beta}{2 \beta} \tanh \left(d_{1}\right) t+\delta\right]$ \\
\hline
\end{tabular}

Table 2

Examples of DDEs and their solutions computed with DDESpecialSolutions.m. 
The main function, DDESpecialSolutions, uses five subroutines corresponding to the five steps of the algorithm.

(1) DDESpecialSolutions VarChange returns a list of nonlinear DDEs in $T_{\mathbf{n}}$.

(2) DDESpecialSolutionsmSolver uses the result from DDESpecialSolutionsVarChange to determine the degree(s), $M_{i}$, of the polynomial solutions.

(3) DDESpecialSolutionsBuildSystem uses the output of DDESpecialSolutions VarChange and DDESpecialSolutionsmSolver to build the nonlinear algebraic system for the $a_{i j}$. The output of DDESpecialSolutionsBuildSystem is a list of sub-lists containing the algebraic system, the unknowns, the wave parameters $\left(d_{i}, c_{j}\right)$, the parameters $(\alpha, \beta, \ldots)$, and the nonzero variables $\left(a_{i, M_{i}}, d_{i}, c_{j}, \alpha, \beta, \ldots\right)$.

(4) Algebra'AnalyzeAndSolve'AnalyzeAndSolve solves the system returned by DDESpecialSolutionsBuildSystem.

(5) DDESpecialSolutionsBuildSolutions builds and tests the solutions using the output from Algebra'AnalyzeAndSolve'AnalyzeAndSolve, DDESpecialSolutionsmSolver, and the original system of DDEs.

\section{Discussion and Conclusions}

We presented a straightforward algorithm to compute special solutions of nonlinear polynomial DDEs, without using explicit integration. We designed the symbolic package DDESpecialSolutions.m to find traveling wave solutions of nonlinear DDEs involving the tanh functions. Our code is designed to handle DDEs with multiple continuous variables and/or multiple lattice points $(n, m, \cdots)$. However, we were unable to find semi-discrete lattices with multiple lattice points in the literature.

While the software reproduces the known solutions for many equations, there is no guarantee that the code will compute the complete solution set of all polynomial solutions involving the tanh function, especially when the DDEs have parameters. This is due to restrictions on the form of the solutions and the limitations of the algebraic solver. Furthermore, the nonlinear constraints which arise in solving the nonlinear algebraic system may be quintic or of higher degree, and therefore unsolvable in analytic form. Also, since our software package is fully automated, it may not return the solutions in the simplest form.

Often, the nonlinear solver returns constraints on the wave parameters $c_{j}$ and the external parameters (lower-case Greek letters), if any. In principle, one should verify whether or not such constraints affect the results of the previous steps in the algorithm. In particular, one should verify the consistency with the results from Step 2 of the algorithm. 
There are several ways our algorithm could be generalized. One could look for solutions other than polynomials in tanh. Rational solutions would be an obvious choice since $T_{\mathbf{n}+\mathbf{p}_{j}}$ is a rational expression in $T_{\mathbf{n}}$. One may try to compute exact solutions in terms of functions $f$ (other than tanh). We restricted our algorithm to tanh solutions because $f\left(\xi_{\mathbf{n}+\mathbf{p}_{j}}\right)=\tanh \left(\xi_{\mathbf{n}+\mathbf{p}_{j}}\right)$ can be expressed in $f\left(\xi_{\mathbf{n}}\right)=\tanh \left(\xi_{\mathbf{n}}\right)$ via

$$
f\left(\xi_{\mathbf{n}+\mathbf{p}_{j}}\right)=\tanh \left(\xi_{\mathbf{n}+\mathbf{p}_{j}}\right)=\frac{\tanh \left(\xi_{\mathbf{n}}\right)+\tanh \left(\phi_{j}\right)}{1+\tanh \left(\xi_{\mathbf{n}}\right) \tanh \left(\phi_{j}\right)}=\frac{f\left(\xi_{\mathbf{n}}\right)+\tanh \left(\phi_{j}\right)}{1+f\left(\xi_{\mathbf{n}}\right) \tanh \left(\phi_{j}\right)},
$$

which allowed us to use chainrule (19). If tanh were replaced by another function $f$, the function must have the property that $f\left(\xi_{\mathbf{n}+\mathbf{p}_{j}}\right)$ can be expressed as some function of $f\left(\xi_{\mathbf{n}}\right)$. Otherwise a modified chain rule for $f$ would no longer work.

\section{Acknowledgements}

This material is based upon work supported by the National Science Foundation (NSF) under Grants Nos. CCR-9901929, DMS-9732069 and DMS9912293. Any opinions, findings, and conclusions or recommendations ex-

pressed in this material are those of the authors and do not necessarily reflect the views of NSF.

WH thankfully acknowledges the hospitality and support of the Department of Applied Mathematics of the University of Stellenbosch, South Africa, during his sabbatical visit in Spring 2001. Part of the work was done at Wolfram Research, Inc., while WH was supported by a Visiting Scholar Grant in Fall 2000 .

The authors thank P. Blanchard, J. Blevins, J. Heath, J. Milwid, and M. Porter-Peden for their help with the project.

\section{Appendix A. Test run description}

We illustrate the use of the package DDESpecialSolutions.m on a PC. Users should have access to Mathematica v. 3.0 or higher.

Put the package in a directory, say myDirectory, on drive C. Start a Mathematica notebook session and execute the commands:

SetDirectory ["c: \\myDirectory"]; (* specify directory *) 


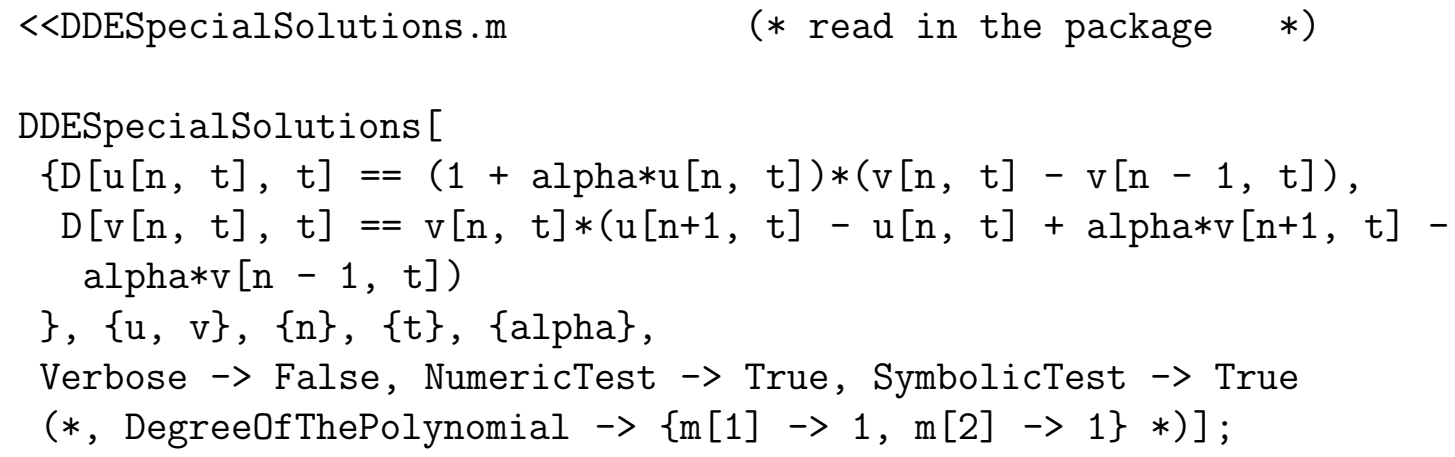

If the DegreeOfThePolynomial $\rightarrow\{m[1] \rightarrow 1, m[2] \rightarrow 1\}$ were specified, the code would continue with this case only and not attempt to compute the degrees of the polynomials $U\left(T_{\mathbf{n}}\right)$ and $V\left(T_{\mathbf{n}}\right)$.

The output of the above is:

DDESpecialSolutionsmSolver:: "remove" :

The potential solutions $\{\{m[1] \rightarrow 1, m[2] \rightarrow 2\}\}$ are being removed because they are (i) negative, (ii) contain freedom, (iii) fail to balance highest exponent terms from two different terms in the original system. If $M_{i}<0$, then the transformation $u \rightarrow 1 / v$ may result in a system that DDESpecialSolutions can solve.

Numerically testing the solutions.

Symbolically testing the solutions.

$$
\begin{gathered}
\left\{\left\{\left\{u(n, t) \rightarrow-\left(\frac{1+\alpha c(2) \operatorname{coth}(c(1))-\alpha c(2) \tanh (\text { phase }+n c(1)+t c(2))}{\alpha}\right),\right.\right.\right. \\
\left.\left.\left.v(n, t) \rightarrow \frac{c(2)(\operatorname{coth}(c(1))-\tanh (\text { phase }+n c(1)+t c(2)))}{\alpha}\right\}\right\}\right\}
\end{gathered}
$$

The package DDESpecialSolutions.m has been tested on various PCs with Mathematica versions 3.0, 4.0, 4.1 and 5.0.

\section{References}

[1] M.J. Ablowitz, J.F. Ladik, J. Math. Phys. 16 (1975) 598-603.

[2] M.J. Ablowitz, J.F. Ladik, Stud. Appl. Math. 55 (1976) 213-229.

[3] M.J. Ablowitz, J.F. Ladik, Stud. Appl. Math. 57 (1977) 1-12.

[4] V.E. Adler, S.I. Svinolupov, R.I. Yamilov, Phys. Lett. A 254 (1999) 24-36. 
[5] V.E. Adler, A.B. Shabat, R.I. Yamilov, Theor. Math. Phys. 125 (2000) 16031661.

[6] D. Baldwin, Ü. Göktaş, W. Hereman, L. Hong, R.S. Martino, J.C. Miller, Symbolic computation of exact solutions expressible in hyperbolic and elliptic functions for nonlinear PDEs, J. Symb. Comp. (2003) in press.

[7] D. Baldwin, Ü. Göktaş, W. Hereman, L. Hong, R.S. Martino, J.C. Miller, PDESpecialSolutions.m: A Mathematica program for the symbolic computation of exact solutions expressible in hyperbolic and elliptic functions for nonlinear PDEs, 2001, http://www.mines.edu/fs_home/whereman/.

[8] D. Baldwin, Ü. Göktaş, W. Hereman, L. Hong, R.S. Martino, J.C. Miller, DDESpecialSolutions.m: A Mathematica program for the symbolic computation of hyperbolic tangent solutions of nonlinear differential-difference equations, 2003, http://www.mines.edu /fs_home/whereman/.

[9] I.Yu. Cherdantsev, R.I. Yamilov, Physica D 87 (1995) 140-144.

[10] I.Yu. Cherdantsev, R. Yamilov, Local master symmetries of differentialdifference equations, in: D. Levi, L. Vinet, P. Winternitz (Eds.), Symmetries and Integrability of Difference Equations, CRM Proc. \& Lect. Notes 9, AMS, Providence, Rhode Island, 1996, pp. 51-61.

[11] E. Fan, J. Phys. A.: Math. Gen. 35 (2002) 6853-6872.

[12] E. Fan, J. Phys. A: Math. Gen. 36 (2003) 7009-7026.

[13] E. Fan, Chaos, Solitons and Fractals 16 (2003) 819-839.

[14] E. Fermi, J. Pasta, S. Ulam, Collected papers of Enrico Fermi II, University of Chicago Press, Chicago, Illinois, 1965, p. 978.

[15] W. Hereman, J.A. Sanders, J. Sayers, J.P. Wang, Symbolic Computation of Conserved Densities, Generalized Symmetries, and Recursion Operators for Nonlinear Differential-Difference Equations, in: D. Levi, P. Winternitz (Eds.), CRM Proc. \& Lect. Notes, AMS, Providence, Rhode Island, 2004, submitted.

[16] M. Hickman, W. Hereman, Proc. Roy. Soc. Lond. A 459 (2203) 2705-2729.

[17] R. Hirota, M. Iwao, Time-discretization of soliton equations, in: D. Levi, O. Ragnisco (Eds.), SIDE III-Symmetries and Integrability of Difference Equations, CRM Proc. \& Lect. Notes 25, AMS, Providence, Rhode Island, 2000, pp. 217-229

[18] M. Kac, P. van Moerbeke, Adv. Math. 16 (1975) 160-169.

[19] K. Kajiwara, J. Satsuma, J. Math Phys. 32 (1991) 506-514.

[20] D. Levi, O. Ragnisco, Lett. Nuovo Cimento 22 (1978) 691-696.

[21] D. Levi, R.I. Yamilov, J. Math. Phys. 38 (1997) 6648-6674.

[22] Z.B. Li \& Y.P. Liu, Comp. Phys. Comm. 148 (2002) 256-266. 
[23] Y.P. Liu, Z.B. Li, Comp. Phys. Comm. 155 (2003) 65-76.

[24] A. Maccari, Nonlinearity 15 (2002) 807-815.

[25] A.V. Mikhailov, A.B. Shabat, R.I. Yamilov, Usp. Mat. Nauk 24 (1987) 3-53; (Engl. Transl. Russian Math. Surveys 42 (1987) 1-63.

[26] A.V. Mikhailov, A.B. Shabat, V.V. Sokolov, The symmetry approach to classification of integrable equations, in: V.E. Zakharov (ed.), What is Integrability?, Springer Verlag, Berlin, 1990, pp. 115-184.

[27] E.J. Parkes, B.R. Duffy, P.C. Abbott, Phys. Lett. A 295 (2002) 280-286.

[28] S.N.M. Ruijsenaars, Comm. Math. Phys. 133 (1990) 217-247.

[29] A.B. Shabat, R.I. Yamilov, Leningrad Math. J. 2 (1991) 377-400.

[30] A.B. Shabat, R.I. Yamilov, Phys. Lett. A 227 (1997) 15-23.

[31] V.V. Sokolov, A.B. Shabat, Sov. Scient. Rev. C; Math. Phys. Rev. 4 (1984) $221-280$.

[32] Yu.B. Suris, J. Phys. A: Math. Gen. 30 (1997) 1745-1761.

[33] Yu.B. Suris, J. Phys. A: Math. Gen. 30 (1997) 2235-2249.

[34] Yu.B. Suris, Miura transformations for Toda-type integrable systems, with applications to the problem of integrable discretizations, Preprint SFB 288, Fachbereich Mathematik, Technische Universität Berlin, Berlin, Germany, 1998.

[35] Yu.B. Suris, Rev. Math. Phys. 11 (1999) 727-822.

[36] Yu.B. Suris, Miura transformations for Toda-type integrable systems, with applications to the problem of integrable discretizations, Sfb288 Preprint 367, Department of Mathematics, Technical University Berlin, Berlin, Germany, 2001.

[37] Yu.B. Suris, The problem of integrable discretization: Hamiltonian approach. A skeleton of the book, Sfb288 Preprint 479, Department of Mathematics, Technical University Berlin, Berlin, Germany, 2002.

[38] S.I. Svinolupov, R.I. Yamilov, Phys. Lett. A 160 (1991) 548-552.

[39] G. Teschl, Jacobi Operators and Completely Integrable Nonlinear Lattices, AMS Mathematical Surveys and Monographs 72, AMS, Providence, Rhode Island, 2000.

[40] M. Toda, Theory of Nonlinear Lattices, Springer Verlag, Berlin, Germany, 1981.

[41] R.I. Yamilov, Classification of Toda type scalar lattices, in: V. Makhankov, I. Puzynin, O. Pashaev (Eds.), Proc. 8th Int. Workshop on Nonlinear Evolution Equations and Dynamical Systems, NEEDS'92, Dubna, U.S.S.R., 1992, World Scientific, Singapore, 1993, pp. 423-431.

[42] R.I. Yamilov, J. Phys. A: Math. Gen. 27 (1994) 6839-6851. 


\begin{tabular}{|c|c|c|c|}
\hline Equation Name & Equations & Solution(s) & Ref. \\
\hline $\begin{array}{l}\text { Ablowitz-Ladik } \\
\text { Lattice }\end{array}$ & $\begin{aligned} \dot{u}_{n}(t) & =\left(\alpha+u_{n} v_{n}\right)\left(u_{n+1}+u_{n-1}\right)-2 \alpha u_{n} \\
\dot{v}_{n}(t) & =-\left(\alpha+u_{n} v_{n}\left(v_{n+1}+v_{n-1}\right)+2 \alpha v_{n}\right.\end{aligned}$ & $\begin{array}{l}u_{n}(t)=\frac{\alpha \sinh ^{2}\left(d_{1}\right)}{a_{21}}\left( \pm 1-\tanh \left[d_{1} n+2 \alpha t \sinh ^{2}\left(d_{1}\right)+\delta\right]\right) \\
v_{n}(t)=a_{21}\left( \pm 1+\tanh \left[d_{1} n+2 \alpha \sinh ^{2}\left(d_{1}\right) t+\delta\right]\right)\end{array}$ & {$[1,2]$} \\
\hline Toda Lattice & $\ddot{u}_{n}(t)=\left(\dot{u}_{n}+1\right)\left(u_{n-1}-2 u_{n}+u_{n+1}\right)$ & $u_{n}(t)=a_{10} \pm \sinh \left(d_{1}\right) \tanh \left[d_{1} n \pm \sinh \left(d_{1}\right) t+\delta\right]$ & {$[40]$} \\
\hline $\begin{array}{l}(2+1) \text { Dimensional } \\
\text { Toda Lattice }\end{array}$ & $\frac{\partial^{2} u_{n}}{\partial x \partial t}(x, t)=\left(\frac{\partial u_{n}}{\partial t}+1\right)\left(u_{n-1}-2 u_{n}+u_{n+1}\right)$ & $u_{n}(x, t)=a_{10}+\frac{1}{c_{2}} \sinh ^{2}\left(d_{1}\right) \tanh \left[d_{1} n+\frac{\sinh ^{2}\left(d_{1}\right)}{c_{2}} x+c_{2} t+\delta\right]$ & [19] \\
\hline $\begin{array}{l}\text { Another } \\
\text { Toda Lattice }\end{array}$ & $\begin{array}{l}\dot{u}_{n}(t)=u_{n}\left(v_{n}-v_{n-1}\right) \\
\dot{v}_{n}(t)=v_{n}\left(u_{n+1}-u_{n}\right)\end{array}$ & $\begin{array}{l}u_{n}(t)=-\operatorname{coth}\left(d_{1}\right) c_{1}+c_{1} \tanh \left(d_{1} n+c_{1} t+\delta\right) \\
v_{n}(t)=-\operatorname{coth}\left(d_{1}\right) c_{1}-c_{1} \tanh \left(d_{1} n+c_{1} t+\delta\right)\end{array}$ & [32] \\
\hline $\begin{array}{l}\text { Relativistic } \\
\text { Toda Lattice }\end{array}$ & $\begin{array}{l}\dot{u}_{n}(t)=\left(1+\alpha u_{n}\right)\left(v_{n}-v_{n-1}\right) \\
\dot{v}_{n}(t)=v_{n}\left(u_{n+1}-u_{n}+\alpha v_{n+1}-\alpha v_{n-1}\right)\end{array}$ & $\begin{array}{l}u_{n}(t)=-\frac{1}{\alpha}-c_{1} \operatorname{coth}\left(d_{1}\right)+c_{1} \tanh \left(d_{1} n+c_{1} t+\delta\right) \\
v_{n}(t)=\frac{c_{1}}{\alpha} \operatorname{coth}\left(d_{1}\right)-\frac{c_{1}}{\alpha} \tanh \left(d_{1} n+c_{1} t+\delta\right)\end{array}$ & {$[34]$} \\
\hline $\begin{array}{l}\text { Another Relativistic } \\
\text { Toda Lattice }\end{array}$ & $\begin{array}{l}\dot{u}_{n}(t)=\left(u_{n+1}-v_{n}\right) v_{n}-\left(u_{n-1}-v_{n-1}\right) v_{n-1} \\
\dot{v}_{n}(t)=v_{n}\left(u_{n+1}-u_{n}\right)\end{array}$ & $\begin{array}{l}u_{n}(t)=\operatorname{coth}\left(d_{1}\right) c_{1}+c_{1} \tanh \left(d_{1} n+c_{1} t+\delta\right) \\
v_{n}(t)=\operatorname{coth}\left(d_{1}\right) c_{1}+c_{1} \tanh \left(d_{1} n+c_{1} t+\delta\right)\end{array}$ & {$[32]$} \\
\hline Volterra Lattice & $\begin{aligned} \dot{u}_{n}(t) & =u_{n}\left(v_{n}-v_{n-1}\right) \\
\dot{v}_{n}(t) & =v_{n}\left(u_{n+1}-u_{n}\right)\end{aligned}$ & $\begin{array}{l}u_{n}(t)=-c_{1} \operatorname{coth}\left(d_{1}\right)+c_{1} \tanh \left(d_{1} n+c_{1} t+\delta\right) \\
v_{n}(t)=-c_{1} \operatorname{coth}\left(d_{1}\right)-c_{1} \tanh \left(d_{1} n+c_{1} t+\delta\right)\end{array}$ & {$[34]$} \\
\hline $\begin{array}{l}\text { Discretized mKdV } \\
\text { Lattice }\end{array}$ & $\dot{u}_{n}(t)=\left(\alpha-u_{n}^{2}\right)\left(u_{n+1}-u_{n-1}\right)$ & $u_{n}(t)= \pm \sqrt{\alpha} \tanh \left(d_{1}\right) \tanh \left[d_{1} n+2 \alpha \tanh \left(d_{1}\right) t+\delta\right]$ & {$[3]$} \\
\hline Hybrid Lattice & $\dot{u}_{n}(t)=\left(1+\alpha u_{n}+\beta u_{n}^{2}\right)\left(u_{n-1}-u_{n+1}\right)$ & $u_{n}(t)=\frac{-\alpha \pm \sqrt{\alpha^{2}-4 \beta} \tanh \left(d_{1}\right)}{2 \beta} \tanh \left[d_{1} n+\frac{\alpha^{2}-4 \beta}{2 \beta} \tanh \left(d_{1}\right) t+\delta\right]$ & [17] \\
\hline
\end{tabular}

Table 3. Alternate version of Table 2. 